\title{
Lipodistrofia Parcial Familiar tipo 2: a propósito de un caso
}

\begin{abstract}
David Araújo-Vilar ${ }^{1,2 *}$, Antía Fernández-Pombo ${ }^{1,2}$, Álvaro Hermida-Ameijeiras ${ }^{1,3}$, Sofía Sánchez-Iglesias ${ }^{1}$
'UETeM-Molecular Pathology Group, IDIS-CIMUS (Centro de Investigación en Medicina Molecular y Enfermedades Crónicas), Universidadede Santiago de Compostela, Galiza. ${ }^{2}$ Servizo de Endocrinoloxía e Nutrición, Complexo Hospitalario Universitario de Santiago, Santiago de Compostela, Galiza. ${ }^{3}$ Servizo de Medicina Interna. Complexo Hospitalario Universitario de Santiago, Santiago de Compostela, Galiza
\end{abstract}

\section{RESUMEN}

Los síndromes de lipodistrofia son trastornos heterogéneos poco frecuentes que se caracterizan por deficiencia de tejido adiposo, generalmente una disminución de los niveles de leptina y, con frecuencia, anomalías metabólicas graves como diabetes mellitus y dislipidemia. El diagnóstico de lipodistrofia se basa en la historia clínica, el examen físico y la evaluación de la composición corporal, siendo útiles los hallazgos de laboratorio en algunos casos.

Describimos el caso de una paciente que presentó varias características fenotípicas habituales de lipodistrofia parcial familiar y complicaciones cardíacas descritas en los pacientes con enfermedad de Dunnigan. Es importante que internistas y cardiólogos consideren la lipodistrofia parcial familiar (FPLD, por sus siglas en inglés) en el diagnóstico diferencial cuando los pacientes con aparente hipertrofia muscular presentan miocardiopatía, resistencia a la insulina, diabetes mellitus y dislipidemia.

Palabras clave: Lipodistrofia, LMNA, laminopatía, diabetes mellitus, resistencia a la insulina

\section{INTRODUCCIÓN}

Las lipodistrofias son un conjunto heterogéneo de enfermedades caracterizadas por una pérdida del tejido adiposo ${ }^{1}$. Con la excepción de la lipodistrofia asociada a la infección por el virus de la inmunodeficiencia humana, el resto son enfermedades sumamente infrecuentes, con una prevalencia estimada entre 1.3 y 4.7 casos por millón de habitantes².

Estas entidades pueden tener un origen genético 0 ser adquiridas $^{1}$, habiéndose descrito más de 40 subtipos diferentes ${ }^{3}$. No obstante, las formas mejor conocidas son cuatro, la lipodistrofia congénita generalizada o síndrome de BerardinelliSeip, la lipodistrofia parcial familiar, la lipodistrofia generalizada adquirida o síndrome de Lawrence y la lipodistrofia parcial adquirida 0 síndrome de Barraquer-Simons ${ }^{1,4}$.

La mayoría de las lipodistrofias se suelen asociar con complicaciones metabólicas (diabetes mellitus no cetósica, hipertrigliceridemia con colesterol-HDL bajo), en el contexto de un cuadro de resistencia grave a la insulina, y hepáticas (esteatosis hepática) ${ }^{1,4}$. Estas complicaciones son las que, en general, van a condicionar el pronóstico de estos pacientes.

La lipodistrofia parcial familiar (LDPF) es con diferencia la más frecuente de las lipodistrofias raras. Se han descrito 9 subtipos dependiendo del gen responsable, pudiendo seguir un patrón de herencia co-dominante, dominante, recesivo 0 poligénico. Aunque entre ellos el subtipo más común es el

\section{ABSTRACT}

Lipodystrophy syndromes are rare heterogeneous disorders characterized by deficiency of adipose tissue, usually a decrease in leptin levels and, frequently, severe metabolic abnormalities including diabetes mellitus and dyslipidemia. Diagnosis of lipodystrophy is based on clinical history, physical examination, and assessment of body composition, with laboratory findings useful in some cases.

This is a case report of a patient presented with several classic features of familial partial lipodystrophy and cardiac complications described in those patients with Dunnigan disease. Familiar partial lipodystrophy (FPLD) is important for the internists and cardiologists to consider in differential diagnosis when apparently muscular looking patients present with cardiomyopathy, insulin resistance, diabetes mellitus, and dyslipidemia.

Key words: Lipodystrophy, LMNA, laminopathies, diabetes mellitus, insulin resistance

síndrome de Köbberling o LDPF tipo $1^{5}$, un trastorno poligénico, el mejor caracterizado es el subtipo 20 enfermedad de Dunnigan 6 .

La enfermedad de Dunnigan está causada por variantes, habitualmente en heterocigosis, en el gen LMNA ${ }^{7}$, que codifica para la proteína lamina $A / C$. Se trata, por tanto, de una laminopatía. La mayoría de las variantes que causan esta enfermedad se localizan entre los exones 8 y 11 de dicho gen, siendo el codón que codifica para la arginina en la posición 482, un "hot spot" de mutagénesis4.

La LDPF tipo 2 se caracteriza por la pérdida de tejido adiposo en extremidades superiores e inferiores y región glúteofemoral, asociada a un acúmulo de grasa en cara, sotabarba, axilas, labios mayores y grasa visceral abdominal. Este fenotipo se hace evidente en las mujeres en la pubertad/adolescencia, mientras que en los hombres suele aparecer más tarde. Además, estas pacientes presentan una musculatura bien definida, con una característica hipertrofia de los gemelos. El árbol venoso en las extremidades suele ser evidente, y con frecuencia presentan acantosis nigricans. En ocasiones pueden presentar lipomas, incluso en áreas lipoatróficas, 10 que puede ayudar a orientar el diagnostico etiológico ${ }^{8}$.

Las complicaciones más frecuentes son la diabetes mellitus no cetósica, la hipertrigliceridemia y la esteatosis hepática. La pancreatitis aguda secundaria a hipertrigliceridemia grave 
puede ser una complicación que comprometa la vida de estos enfermos. La enfermedad cardiovascular aterosclerosa es más frecuente en estos pacientes, sobre todo en las mujeres ${ }^{9}$. Así mismo, también es habitual que estas pacientes presenten trastornos en la esfera reproductiva y síndrome del ovario poliquístico. En raras ocasiones pueden aparecer cuadros de solapamiento con otras laminopatías, principalmente miocardiopatía hipertrófica, valvulopatías, trastornos del ritmo cardíaco y miopatías ${ }^{10}$.

\section{CASO CLÍNICO}

Tanto la exposición de datos clínicos como de las imágenes que se muestran fueron autorizados de forma expresa por la paciente.

Mujer de 48 años, diagnosticada de diabetes mellitus a los 34 y que consulta por mal control metabólico. A la paciente se le habían extirpado varios lipomas en la base del cuello y en la axila izquierda.

La paciente refería episodios de dolor precordial de características opresivas durante el ejercicio, palpitaciones en reposo, hiperfagia, claudicación intermitente a 100 metros y dolores musculares generalizados. Recibía tratamiento con metformina (850 mg bid), sitagliptina (100 mg qd), gliclacida (120 mg qd), pravastatina (40 mg qd), fenofibrato (160 mg qd) e insulina glargina (30 IU qd). La paciente no presentaba complicaciones microangiopáticas de la diabetes. En la exploración física, la paciente pesaba $57 \mathrm{~kg}$ y medía $150 \mathrm{~cm}$ (IMC: $25.3 \mathrm{~kg} / \mathrm{m}^{2}$ ), siendo su presión arterial de 140/81 mmHg. La paciente presentaba un fenotipo cushingoide, con acúmulo de grasa en cara, cuello, cintura escapular, axilas, parte superior de la espalda y monte de Venus. Por otra parte, presentaba una marcada lipoatrofia en extremidades superiores e inferiores y región glúteo-femoral, conservando la grasa en palmas y plantas (Figura 1). Así mismo, se objetivó acantosis nigricans en cuello, axilas e ingles, y una mínima flebomegalia en las extremidades inferiores. La paciente refirió que este fenotipo se inició durante la adolescencia y que era similar al que presentaban sus hermanas, su madre y una tía materna. Presentaba además múltiples lipomas en tórax, axilas y extremidades superiores, así como una hepatomegalia de 6 $\mathrm{cm}$ bajo la arcada costal. El resto de la exploración física fue anodina. Entre las pruebas complementarias que se realizaron destacaron: HbA1c: 8.9\%, colesterol-LDL: $164 \mathrm{mg} / \mathrm{dL}$, colesterol-HDL: $34 \mathrm{mg} / \mathrm{dL}$, triglicéridos: $224 \mathrm{mg} / \mathrm{dL}$, leptina: $2.9 \mathrm{ng} / \mathrm{mL}$ y péptido C: $1.6 \mathrm{ng} / \mathrm{mL}$. La ecografía abdominal reveló una esteatosis hepática, siendo el ecocardiograma normal. La ergometría fue positiva para cardiopatía isquémica, y la angiografía coronaria reveló enfermedad de un vaso colocándose stent farmacoactivo.

Ante la sospecha clínica de lipodistrofia parcial familiar se realizaron mediciones antropométricas y estudio de la composición corporal mediante DXA (absorciometría de rayos $X$ de energía dual) (Figura 2). Pliegues cutáneos: tricipital: 4 mm,
Figura 1. Paciente con lipodistrofia parcial familiar tipo 2. Se puede apreciar la ausencia de tejido adiposo en extremidades y región glúteo-femoral, acúmulo de grasa en cara, cuello, cintura escapular y axilas, así como una musculatura marcada, principalmente en nalgas y extremidades inferiores.

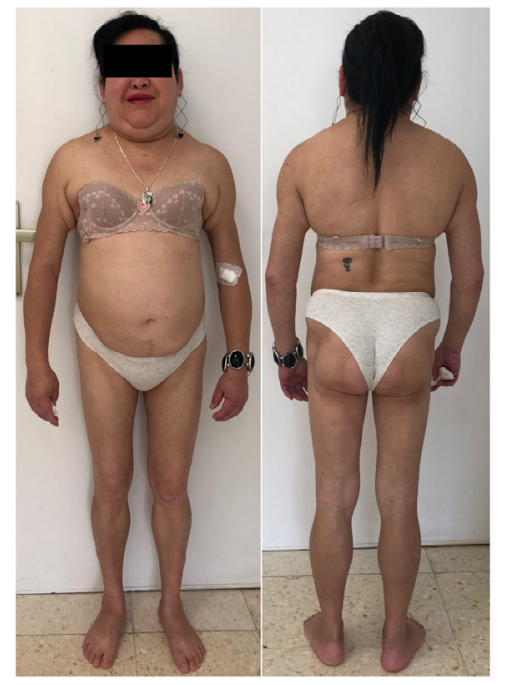

Figura 2. DXA. Panel A: paciente descrita en el caso clínico. Panel B: control sano con un IMC semejante. Las áreas rojas y amarillas representan acúmulo de tejido adiposo, mientras que las áreas verdes representa zonas con escasa cantidad de grasa.

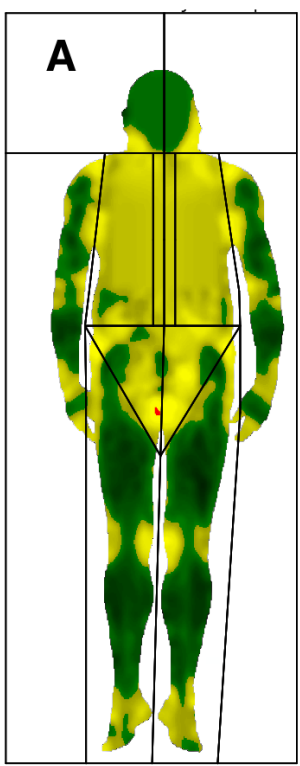

Caso clínico

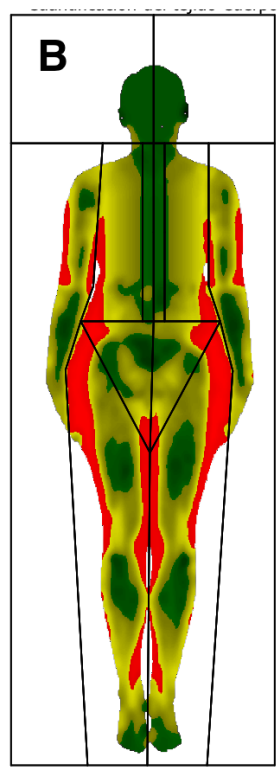

Control bicipital: $3.5 \mathrm{~mm}$, suparilíaco: $8 \mathrm{~mm}$, subescapular: $40 \mathrm{~mm}$, muslo: $4 \mathrm{~mm}$, pantorrilla: $4 \mathrm{~mm}$.

Composición corporal: grasa total: $28,1 \%$, grasa brazos: $24.6 \%$, grasa piernas: $18.3 \%$, grasa tronco: $34.8 \%$.

Dada la presencia de lipomas y los antecedents familiares se procedió directamente a la secuenciación por Sanger del gen LMNA, identificando la variante c.1444C>T, p.(Arg482Trp), en heterocigosis, confirmando el diagnóstico de lipodistrofia parcial familiar tipo 2. 


\section{DISCUSIÓN}

La LDPF es una entidad clínica poco frecuente y por lo tanto mal conocida. Esto hace que muy habitualmente o no se diagnostique o se etiquete a los pacientes por las comorbilidades asociadas: diabetes mellitus (erróneamente tipo 2), dislipemia o hígado graso. Como en el caso clínico descrito. Sin embargo, al menos en su presentación clásica, el fenotipo resulta muy obvio en las mujeres; no así en los hombres que, por norma general, se diagnostican a partir de las mujeres de la familia.

El diagnóstico diferencial incluye el síndrome de Cushing, fundamentalmente por la asociación de acúmulo de grasa en cara y sotabarba, hirsutismo y alteraciones en el metabolismo de la glucosa. Sin embargo, ni la pérdida de tejido adiposo ${ }^{11} \mathrm{ni}$ la hipermuscularidad son características del hipercortisolismo crónico.

La LDPF puede ser una enfermedad mendeliana, asociada a los genes LMNA, PPARG, PLIN1, LIPE, AKT2, ADRA2A,CIDEC, PCYT1Ao MFN2, o poligénica (LDPF tipo $1)^{3,4}$. Las diferencias entre estas entidades suelen ser sutiles y se escapan del análisis de esta Nota Clínica.

En general, ante la sospecha de una lipodistrofia, y sobre todo si se piensa en un subtipo de causa genética, deben realizarse los correspondientes estudios moleculares. Aunque en el caso descrito las características clínicas orientaban claramente a una variante en el gen LMNA, por lo que se procedió a la secuenciación por el método Sanger de dicho gen, Io más eficiente es solicitar un panel NGS especifico para lipodistrofias como el que ha desarrollado la Fundación Galega de Medicina Xenómica.

El diagnóstico temprano de la LDPF resulta crítico, no sólo porque permite reducir el riesgo de desarrollar comorbilidades, sino porque al tratarse de trastornos mendelianos permitiría proporcionar consejo genético que debería incluir información sobre el diagnóstico pre-implantacional a las mujeres con intención de gestar. Además, principalmente en los subtipos autosómicos dominantes, la confirmación molecular del diagnóstico dará pie a la búsqueda de otros casos en un determinado pedigrí.

Con el fin de contribuir a facilitar el diagnóstico de las lipodistrofias infrecuentes, nuestro grupo ha desarrollado un algoritmo diagnóstico que ha implementado en una aplicación (app) gratuita para móviles (LipoDDx $\left.{ }^{\circledR}\right)$, disponible para Android (https://play.google.com/store/apps/details?id=araujo. lipoddx) e IOS (https://apps.apple.com/es/app/lipoddx/ id1474797838). Esta aplicación ha sido validada, obteniendo un porcentaje de eficacia del 79\%12.

Las lipodistrofias no tienen cura y el tratamiento es el de sus complicaciones $^{1,4}$. La base del tratamiento es la dieta y el ejercicio físico.

En el caso de la enfermedad de Dunnigan, al tratarse de una laminopatía que puede asociarse con miocardiopatías estructurales o alteraciones del ritmo cardíaco, siempre es recomendable un estudio cardiológico minucioso antes de aconsejar una actividad física intensa.

Al no existir estudios reglados, el tratamiento de la diabetes es el estándar para la diabetes mellitus tipo 2, siendo la metformina el fármaco de primera elección, pudiendo resultar útil la pioglitazona'. En algunos pacientes con resistencia a la insulina grave será necesario emplear dosis altas de insulina. La guía multisociedad para el diagnóstico y tratamiento de las lipodistrofias ${ }^{1}$ recomienda utilizar las estatinas como fármacos de primera elección en el tratamiento de la dislipemia. Los fibratos y/o ácidos grasos omega-3 deberán usarse si los triglicéridos son mayores de $500 \mathrm{mg} / \mathrm{dL}$, y se podrían considerar con triglicéridos mayores de $200 \mathrm{mg} / \mathrm{dL}$. El uso de estrógenos orales como anticonceptivos está contraindicado por el riesgo de empeorar la hipertrigliceridemia.

En 2018, la Agencia Europea del Medicamento autorizó la leptina recombinante humana (metreleptin) asociada a la dieta para el tratamiento de las lipodistrofias raras. En el caso de las lipodistrofias parciales, la metreleptina estaría indicada en mayores de 12 años en los que el tratamiento convencional no permita alcanzar un control metabólico adecuado $0^{13}$.

\section{BIBLIOGRAFÍA}

1. Brown RJ, Araujo-Vilar D, Cheung PT, Dunger D, Garg A, Jack M et al. The diagnosis and management of lipodystrophy syndromes: A multi-society practice guideline.J Clin Endocrinol Metab. 2016 Oct; 101:4500-4511.

2. Chiquette E, Oral EA, Garg A, Araújo-Vilar D, Dhankhar P. Estimating the prevalence of generalized and partial lipodystrophy: findings and challenges. Diabetes Metab Syndr Obes. 2017 Sep;10:375-383.

3. von Schnurbein J, Adams C, Akinci B, Ceccarini G, D'Apice MR, Gambineri A et al.European lipodystrophy registry: background and structure.Orphanet J Rare Dis. 2020 Apr;15:17.

4. Araújo-Vilar D, Santini F. Diagnosis and treatment of lipodystrophy: a step-bystep approach.J Endocrinol Invest. 2019 Jan;42(1):61-73.

5. Guillín-Amarelle C, Sánchez-Iglesias S, Castro-Pais A, Rodriguez-Cañete L, Ordóñez-Mayán L, Pazos M et al. Type 1 familial partial lipodystrophy: understanding the Köbberling syndrome.Endocrine. 2016 Jul;54(2):411-421.

6. Garg A. Clinical review\#: Lipodystrophies: genetic and acquired body fat disorders. J Clin Endocrinol Metab. 2011 Nov;96:3313-25.

7. Cao H, Hegele RA. Nuclear lamin A/C R482Q mutation in canadian kindreds with Dunnigan-type familial partial lipodystrophy.Hum Mol Genet. 2000 Jan;9:109-12.

8. Araújo-Vilar D, Victoria B, González-Méndez B, Barreiro F, Fernández-Rodríguez $B$, Cereijo R et al. Histological and molecular features of lipomatous and nonlipomatous adipose tissue in familial partial lipodystrophy caused by LMNA mutations.Clin Endocrinol (0xf). 2012 Jun; 76:816-24.

9. Hegele RA. Premature atherosclerosis associated with monogenic insulinresistance. Circulation. 2001 May;103: 2225-9.

10. Guillín-Amarelle C, Fernández-Pombo A, Sánchez-Iglesias S, Araújo-Vilar D. Lipodystrophic laminopathies: Diagnostic clues. Nucleus. 2018 Mar;9:249-260.

11. Rockall AG, Sohaib SA, Evans D, Kaltsas G, Isidori AM, Monson JP et al. Computed tomography assessment of fat distribution in male and female patients with Cushing's syndrome. Eur J Endocrinol. 2003 Dec; 149:561-567.

12. Araújo-Vilar D, Fernández-Pombo A, Rodríguez-Carnero G, Martínez-Olmos MÁ, Cantón A, Villar-Taibo $R$ et al. LipoDDx: a mobile application for identification of rare lipodystrophy syndromes.Orphanet J Rare Dis. 2020 Sep;15:81.

13. Oral EA, Gorden P, Cochran E, Araújo-Vilar D, Savage DB, Long A et al. Longterm effectiveness and safety of metreleptin in the treatment of patients with partial lipodystrophy.Endocrine. 2019 Jan;64:500-511.

\section{Conflicto de intereses}

D.A.V. ha recibido honorarios en concepto conferencias y asesoría científica de Amryt Pharma y Aegerion Pharma. El resto de los autores declaran no tener conflicto de intereses. 